\title{
RESEARCH
}

\section{KAT5 promotes invasion and metastasis through C-MYC stabilization in ATC}

\author{
Xi Wei1,*, Shang Cai2,3,*, Rebecca J Boohaker², Joshua Fried², Ying Li4, Linfei Hu5, Yi Pan5, Ruifen Cheng5, \\ Sheng Zhang ${ }^{1}$, Ye Tian ${ }^{3}$, Ming Gao ${ }^{5}$ and Bo Xu' ${ }^{2,6}$
}

1Department of Diagnostic and Therapeutic Ultrasonography, Tianjin Medical University Cancer Institute and Hospital, National Clinical Research Center of Cancer, Key Laboratory of Cancer Prevention and Therapy, Tianjin, China

2Department of Oncology, Southern Research Institute and Cancer Cell Biology Program, the University of Alabama at Birmingham Comprehensive Cancer Center, Birmingham, Alabama, USA

3Department of Radiotherapy and Oncology, the Second Affiliated Hospital of Soochow University, Suzhou, China

4The Third Department of Breast Cancer, Tianjin Medical University Cancer Institute and Hospital, National Clinical Research Center of Cancer, Key Laboratory of Cancer Prevention and Therapy, Tianjin, China

${ }^{5}$ Department of Thyroid Tumor, Tianjin Medical University Cancer Institute and Hospital, National Clinical Research Center of Cancer, Key Laboratory of Cancer Prevention and Therapy, Tianjin, China

${ }^{6}$ Department of Molecular Radiation Oncology, Key Laboratory of Breast Cancer Prevention and Therapy, Ministry of Education, National Clinical Research Center of Cancer, Tianjin Medical University Cancer Institute and Hospital, Tianjin, China

Correspondence should be addressed to B Xu or M Gao: bxu@southernresearch.org or xubo@tmu.edu.cn or gaoming@tjmuch.com *(X Wei and S Cai contributed equally to this work)

\begin{abstract}
Anaplastic thyroid cancer (ATC) is an aggressive cancer with poor clinical prognosis. However, mechanisms driving ATC aggressiveness is not well known. Components of the DNA damage response (DDR) are frequently found mutated or aberrantly expressed in ATC. The goal of this study is to establish the functional link between histone acetyltransferase lysine (K) acetyltransferase 5 (KAT5, a critical DDR protein) and ATC invasiveness using clinical, in vitro and in vivo models. We analyzed the expression of KAT5 by immunohistochemistry and assessed its relationship with metastasis and overall survival in 82 ATC patients. Using cellular models, we established functional connection of KAT5 expression and C-MYC stabilization. We then studied the impact of genetically modified KAT5 expression on ATC metastasis in nude mice. In clinical samples, there is a strong correlation of KAT5 expression with ATC metastasis $(P=0.0009)$ and overall survival $(P=0.0017)$. At the cellular level, upregulation of KAT5 significantly promotes thyroid cancer cell proliferation and invasion. We also find that KAT5 enhances the C-MYC protein level by inhibiting ubiquitin-mediated degradation. Further evidence reveals that KAT5 acetylates and stabilizes C-MYC. Finally, we prove that altered KAT5 expression influences ATC lung metastases in vivo. KAT5 promotes ATC invasion and metastases through stabilization of C-MYC, demonstrating it as a new biomarker and therapeutic target for ATC.
\end{abstract}

$\begin{aligned} & \text { Key Words } \\ & \text { - } \text { anaplastic thyroid cancer } \\ & \text { - C-MYC } \\ & \text { - KAT5 } \\ & \text { - invasion } \\ & \text { - } \text { metastasis }\end{aligned}$

\section{Introduction}

Highly aggressive, anaplastic thyroid cancer (ATC) is a rare form of thyroid cancer with median survival of only $\sim 4$ months due to invasive nature and metastatic capacity (Xing 2013, Molinaro et al. 2017). ATC is typically resistant to multimodality therapies such as radiotherapy and chemotherapy, leaving it a continuous (c) 2019 Society for Endocrinology Published by Bioscientifica Ltd. Printed in Great Britain 
challenge to successful treatment. Morphologically, ATC is characterized by multiple atypical mitotic nuclei and osteoclast-like multinuclear giant cells. During thyroid cancer evolution, the process from differentiated to undifferentiated thyroid carcinoma undergoes molecular and genetic alterations (Kitahara \& Sosa 2016). Multiple specific genetic mutations have been identified, such as BRAF, RAS, TERT, PIK3CA, PTEN and ALK genes in ATC (Guerra et al. 2013). Because of the rapid progression and high invasiveness nature of ATC, at the time of diagnosis, most patients have already contracted extra-thyroid invasion and/or lymph metastasis, and many of them have distant metastases (Smallridge et al. 2009). Therefore, there is an urgent need to understand the molecular mechanism(s) driving ATC aggressiveness and to identify novel targets to improving the treatment outcome.

Genetic instability is one of the hallmarks of cancers and may be associated with the underlying cause for ATC aggressiveness (Hanahan \& Weinberg 2011). The DNA damage response (DDR), a major mechanism for the maintenance of genome stability, contains multiple signal transduction pathways mediating cell cycle checkpoints, DNA repair and programmed cell death (Lord \& Ashworth 2012). Abnormal DDR pathways have been documented in the majority of cancers, including ATC (Molinaro et al. 2017). It is widely accepted that optimal DDR mechanisms play an essential role in prevention of tumorigenesis in the early stage of tumor development (Bartek et al. 2007b). Growing evidence has also proved that hyperactivation of the DDR cascade also contributes to tumor progression and metastasis (Boohaker \& $\mathrm{Xu}$ 2014). One critical element in the DDR pathway is lysine (K) acetyltransferase 5 (KAT5), which belongs to the MYST family of histone acetyltransferase (Sun et al. 2010). Also known as Tip60, KAT5 is considered a tumor suppressor gene (Gorrini et al. 2007), where it contributes to an important enzyme in repairing DNA and returning cellular function to normal through its regulation of the ataxia telangiectasia mutant (ATM) protein kinase (Sun et al. 2005, 2007, 2009, Bhoumik et al. 2008, Kaidi \& Jackson 2013). The role of KAT5 in cancer progression is less known. Recent studies have shown that KAT5 is linked to malignancy in some cancers, such as pleural mesothelioma and prostate cancer (Shiota et al. 2010, Cregan et al. 2016) and might serve as a potential drug target in human breast cancer and glioblastoma (Pandey et al. 2015, Sun et al. 2015, Takino et al. 2016).

Due to the rarity of ATC, most of the clinical studies investigating the impact of genetic alterations are limited by sample size, making it difficult to draw clinical conclusions. In this study, we analyzed samples from 82 ATC patients in Tianjin Cancer Hospital and found a strong correlation of KAT5 overexpression and poor clinical prognosis. KAT5 promotes ATC cell invasion and proliferation through acetylating C-MYC, thus inhibiting ubiquitination proteasome degradation and stabilizing the protein. Furthermore, we demonstrate evidence that overexpression of KAT5 promotes metastases in an ATC xenograft model.

\section{Methods and materials}

\section{Patient information and database analysis}

A total of 82 patients diagnosed with ATC between 2001 and 2013 in Tianjin Medical University Cancer Hospital were enrolled in the study. Evaluation of the clinical cases was approved by the Tianjin Medical University Institutional Cancer Hospital Review Board. The average age is 61.8 years (range from 17 to 89 years old). The mean follow-up time is 61 months. The overall survival (OS) time ranges from 19 days to 44 months and the median OS is 6 months. All human tumor tissues were obtained with written informed consent, prior to participation in the study. In the process of identifying potential DDR genes in ATC that might be associated with the clinical aggressiveness, we analyzed the Cancer Genome Atlas (TCGA) database and found a cluster of genes affected in the DDR pathway.

\section{Tissue selection and immunohistochemistry}

Two experienced pathologists (P Y and Ruifen Cheng) reviewed hematoxylin-eosin (HE) staining slides to decide which tissue is representative for ATC. Decided by microscopic findings, the criterion was that selected tissue blocks should contain more tumor tissues, less stromal components, less necrosis and less hemorrhage.

Sections of formalin-fixed tissues from all cases were performed using a standard immunohistochemistry protocol (Wei et al. 2014). Briefly, the $3 \mathrm{~mm}$ tissue sections on coated slides were heated for antigen retrieval, pretreated with a $3 \%$ solution of hydrogen peroxide for 5-10 min, rinsed and incubated with $10 \%$ bovine serum albumin (BSA) as a blocking agent. The sections were incubated with rabbit anti-KAT5 antibody (1:100; Cell Signaling) for $1 \mathrm{~h}$ at room temperature. All steps were preceded by rinsing of sections with PBS ( $\mathrm{pH}$ 7.6). The chromogen was 3,3-diaminobenzidine (DAB). The immunoreaction for KAT5 in the nucleus of tumor cells was (c) 2019 Society for Endocrinology Published by Bioscientifica Ltd. Printed in Great Britain 
evaluated independently by two experienced pathologists and scored by the H-score method that combines the values of immunoreaction intensity and the percentage of tumor cell stained as described (Abdulghani et al. 2016), $(-)=$ no positive cells, $(+)=1-10 \%$ of the cells stained, $(++)=11-50 \%$ of the cells stained and $(+++)=51-100 \%$ of the cells stained.

\section{Cell culture and reagents}

The human papillary thyroid carcinoma cell line TPC1 was generously provided by Dr. Michael Mingzhao Xing (Johns Hopkins University School of Medicine, USA). Human anaplastic thyroid carcinoma cell line 8505C was purchased from Sigma-Aldrich. Both cells were cultured in MEM medium supplemented with 10\% FBS, 1\% penicillin and streptomycin, 1\% NEAA (100X), 1\% sodium pyruvate (100X) and grown in a humidified incubator at $3-7^{\circ} \mathrm{C}$ with $5 \% \mathrm{CO}_{2}$. All tissue culture reagents were purchased from Sigma-Aldrich.

\section{Plasmid, siRNA, shRNA and transfection}

The KAT5-pcDNA HisMax plasmid was kindly provided by Prof. Yuet-kin Leung (University of Cincinnati College of Medicine, USA), and CalFectin (SignaGen, Ijamsville, MD, USA) was used to transfect the plasmid into cells. KAT5 Trilencer-27 siRNA was purchased from OriGene (Rockville). The siRNA and shRNA transfection reagent was purchased from Santa Cruz.

\section{Quantitative reverse transcription PCR}

Total RNAs were extracted from TPC1 and 8505C cell lines by using TRIzol LS reagent (Invitrogen) according to manufacturer's instruction. RNA concentrations were measured by NanoDrop 2000, $2 \mu \mathrm{g}$ RNA was used for reverse transcription by using High-Capacity cDNA reverse transcription kits (Applied Biosystems, Thermo Fisher Scientific). The cDNA is then used as a template for exponential amplification using SYBR Green/ROX qPCR Master Mix (Thermo Fisher Scientific) as follows: $50^{\circ} \mathrm{C}$ for $2 \mathrm{~min}, 95^{\circ} \mathrm{C}$ for $10 \mathrm{~min}$, then 40 cycles of $95^{\circ} \mathrm{C}$ followed by $60^{\circ} \mathrm{C}$ for $1 \mathrm{~min}$. Primer sequences are $C-M Y C$ forward primer: 5'-GCC ACG TCT CCA CAC ATC AG-3'; C-MYC reverse primer: $5^{\prime}$-TCT TGG CAG CAG GAT AGT CCT T-3'; KAT5 forward primer: 5'- AAT GTG GCC TGC ATC CTA AC-3'; KAT5 reverse primer: 5'-TGT TTT CCC TTC CAC TTT GG-3'; GAPDH forward primer: 5'-CGA
CCA CTT TGT CAA GCT CA-3'; GAPDH reverse primer: 5'- AGG GGT CTA CAT GGC AAC TG-3'.

\section{Western blotting}

After treatment, cells were harvested and whole cell lysates were extracted using a RIPA lysis buffer $(50 \mathrm{mM}$ pH7.5 Tris, $1 \mathrm{mM}$ EDTA, $150 \mathrm{mM} \mathrm{NaCl}, 1 \%$ NP40, $0.25 \%$ sodium deoxycholate, protease inhibitor cocktail). The nuclear fraction was extracted using NE-PER nuclear and cytoplasmic extraction reagent (Thermo Fisher Scientific). Equal amounts of protein were separated by SDS-PAGE and transferred to nitrocellulose membranes (Millipore). After blocking for $1 \mathrm{~h}$ with $10 \%$ nonfat milk in TBS-Tween-20 at room temperature, membranes were incubated overnight with primary antibody at $4^{\circ} \mathrm{C}$, washed and incubated with HRP-conjugated secondary antibody (Santa Cruz Technology) for $2 \mathrm{~h}$ at room temperature, and then membranes were washed and visualized by using enhanced chemiluminescence (ECL, Thermo Fisher Scientific).

\section{Cell proliferation}

8505C and TPC1 cells were seeded into 24-well tissue culture plates and incubated for indicated times. Cells were then washed and fixed by crystal violet staining solution. The staining was dissolved into $10 \%$ glacial acetic acid solution and the OD values were detected under $490 \mathrm{~nm}$ wavelength.

\section{Colony formation assay}

8505C and TPC1 cells were seeded into six-well tissue culture plates and incubated overnight. Then, cells were irradiated for indicated doses and continue to incubate for about 2 weeks. Further, the colonies were washed, fixed, stained and counted.

\section{MTT assay}

8505C and TPC1 cells were seeded into 96-well tissue culture plates and incubated overnight. Then, cells were treated with different concentrations of doxorubicin for indicated times. Twenty microliters of MTT solution were then added into each well and incubated for another hour, followed by DMSO dissolving and OD detection under $490 \mathrm{~nm}$ wavelength. (c) 2019 Society for Endocrinology Published by Bioscientifica Ltd. Printed in Great Britain 


\section{Immunoprecipitation assay}

Immunoprecipitation assay was performed by using ImmunoCruz IP/WB system (Santa Cruz Technology). Briefly, cell lysates were suspended into preclearing matrix, rotated and centrifuged. Meanwhile, IP matrix was suspended into PBS with IP antibody and was rotated overnight to form the IP antibody-IP matrix complex. The supernatants were then mixed with the IP antibodyIP matrix complex and rotated overnight. The samples were eventually washed, centrifuged, resuspended, boiled and electrophoresed.

\section{Transwell invasion assay}

8505C and TPC1 cells $\left(2.5 \times 10^{4}\right)$ in FBS-free medium were seeded into the upper chamber coated with Corning Matrigel Matrix of a 24-well plate that contained media with 10\% FBS. After further incubation for indicated times, the cells on the lower side of the chamber were fixed, stained and photographed.

\section{Lung metastatic nude mouse model}

The experimental protocol in nude mice was approved by the Ethics Committee of the Tianjin Medical University Cancer Hospital. All procedures involving animals and their care were conducted in conformity with the institutional guidelines that are in compliance with national and international laws and policies. Male BALB/c nude mice (6-8 weeks old) were purchased from Tianjin Institute of Health and Environmental Medicine. Isogenic TPC1 and 8505C cell lines stably expressing vectoronly, the KAT5 (in TPC1), the KAT5 siRNA (in 8505C) and PBS (as blank control) were injected into the mice (eight for each group) via the tail vein at a concentration of $2 \times 10^{6}$ cells/mouse. At the final time point (10 weeks), mice were killed and lungs were removed and fixed by paraformaldehyde. The lung metastatic foci were detected and calculated macroscopically by three different observers. The representative HE staining nodule slides have reviewed by experienced pathologists.

\section{Statistics analysis}

Data were determined by Student's $t$-test, $\chi^{2}$ test or Pearson correlation test with SPSS 23.0 software (IBM company). The time to death (or confirmed metastasis) or the end of follow-up was calculated for survival analysis. The Kaplan-Meier survival curves were constructed, and between-group differences were tested using the log-rank test and $P \leq 0.05$ was considered significantly.

\section{Results}

\section{KAT5 is overexpressed in ATC and correlated with poor prognosis}

In the process of identifying potential DDR genes in ATC that might be associated with the clinical aggressiveness,

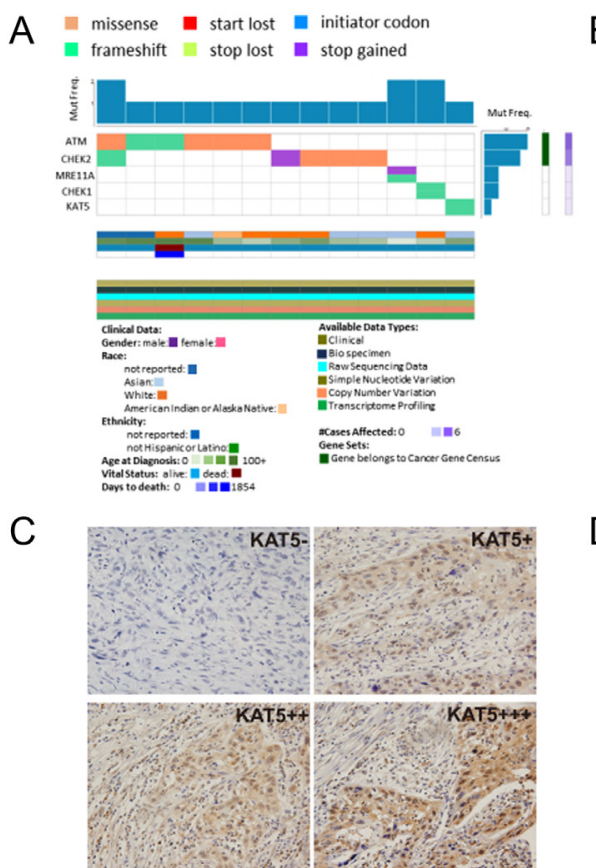

https://erc.bioscientifica.com

https://doi.org/10.1530/ERC-18-0193
B

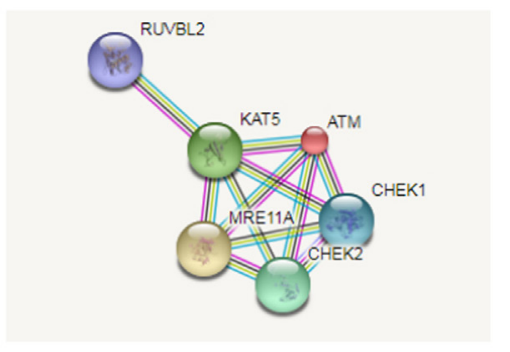

Figure 1

Elevated KAT5 expression correlates with poor survival in patients with ATC. (A) The Cancer Genome Atlas (TCGA) database was analyzed with a cluster of genes affected in the DDR pathway with frameshift or missense somatic mutations, including KAT5, ATM, CHEK2, CHEK1, MRE11A and RUVBL2. (B) The protein networks from String Database were analyzed which connect the DDR proteins with KAT5. (C) The immunoreaction for KAT5 in the nucleus of tumor cells was scored by the $\mathrm{H}$-score method that combines the values of immunoreaction intensity and the percentage of tumor cell stained: $(-)=$ no positive cells, $(+)=1-10 \%$ of the cells stained, $(++)=11-50 \%$ of the cells stained, and $(+++)=51-100 \%$ of the cells stained. (D) Kaplan-Meier curve was performed to analyze the correlation between KAT5 expression and overall survival rate of patients with ATC. 
we analyzed the Cancer Genome Atlas (TCGA) database and found a cluster of genes affected in the DDR pathway (Fig. 1A). Of them, ATM and CHEK2 showed prevalent frameshift or missense somatic mutations. Also found were mutations in CHEK1, MRE11A and RUVBL2. Running these proteins through the String Database (www.string-db.org) for protein networks, we found that these proteins were all integrally connected by KAT5, suggesting that KAT5 might be the center protein associated with altered DDR activities in ATC (Fig. 1B). To validate this notion, we obtained 82 ATC and 45 PTC samples from this cancer center and conducted immunohistochemistry with an anti-KAT5 antibody (Fig. 1C). We found a strong positive correlation of KAT5 expression with ATC metastasis $(P=0.0009$, Table 1$)$. Furthermore, elevated KAT5 expression was associated with poorer overall survival, compared to lower KAT5 expression cases (MST: 8/4 vs 20/12 months, $P=0.0017$, Fig. 1D). We also assessed KAT5 expression in 45 PTC cases, and we found significantly higher expression in ATC than that in PTC $\left(\chi^{2}=14.88, P=0.0019\right)$ (Supplementary Fig. 1A, see section on supplementary data given at the end of this article). Furthermore, we found in the TCGA database that patients with papillary thyroid carcinoma and follicular thyroid carcinoma (PTC and FTC) with KAT5 elevation had worse prognosis than those with normal KAT5 expression $(P=0.0454$, Supplementary Fig. $1 \mathrm{~B}$ and C). Together, we conclude that elevated KAT5 is associated with ATC metastasis and elevated expression is associated with poor prognosis in thyroid carcinoma.
Table 1 Correlation of KAT5 expression and ATC metastasis.

\begin{tabular}{|c|c|c|c|}
\hline KAT5 & Metastasis & Non-metastasis & Pvalue \\
\hline Negative & 2 & 5 & 0.0009 \\
\hline Positive (+) & 23 & 4 & \\
\hline Positive $(++)$ & 31 & 1 & \\
\hline Positive $(+++)$ & 15 & 1 & \\
\hline Total & 71 & 11 & \\
\hline
\end{tabular}

\section{Genetical alteration of KAT5 expression promotes thyroid cancer cell proliferation}

To study the impact of elevated KAT expression in thyroid cancer progression and metastasis, we compared endogenous KAT5 expression in a panel of TC cell lines. The results showed that TPC1 (a PTC cell line) had lowest KAT5 expression and 8505C (an ATC cell line) with the highest in (Supplementary Fig. 2). This prompted us to choose these two cell lines for further studies. Both KAT5 protein and mRNA levels are much higher in $8505 \mathrm{C}$ cells than in TPC1 cells (Fig. 2A). We expressed exogenous KAT5 in TPC1 and 8505C cells (Fig. 2B, lower panel). Crystal violet staining (Fig. 2B upper panel) and colony formation assay (Fig. 2B middle panel) were performed to evaluate the effect of expression of KAT5 on proliferation. We found that upregulation of KAT5 significantly promoted thyroid cancer cell proliferation in both $8505 \mathrm{C}$ and TPC1 cells. Downregulation of KAT5 by siRNA in $8505 \mathrm{C}$ cells significantly reduced the growth of $8505 \mathrm{C}$ cells (Fig. 2C). Together, we conclude that KAT5 expression is associated with thyroid cancer growth.
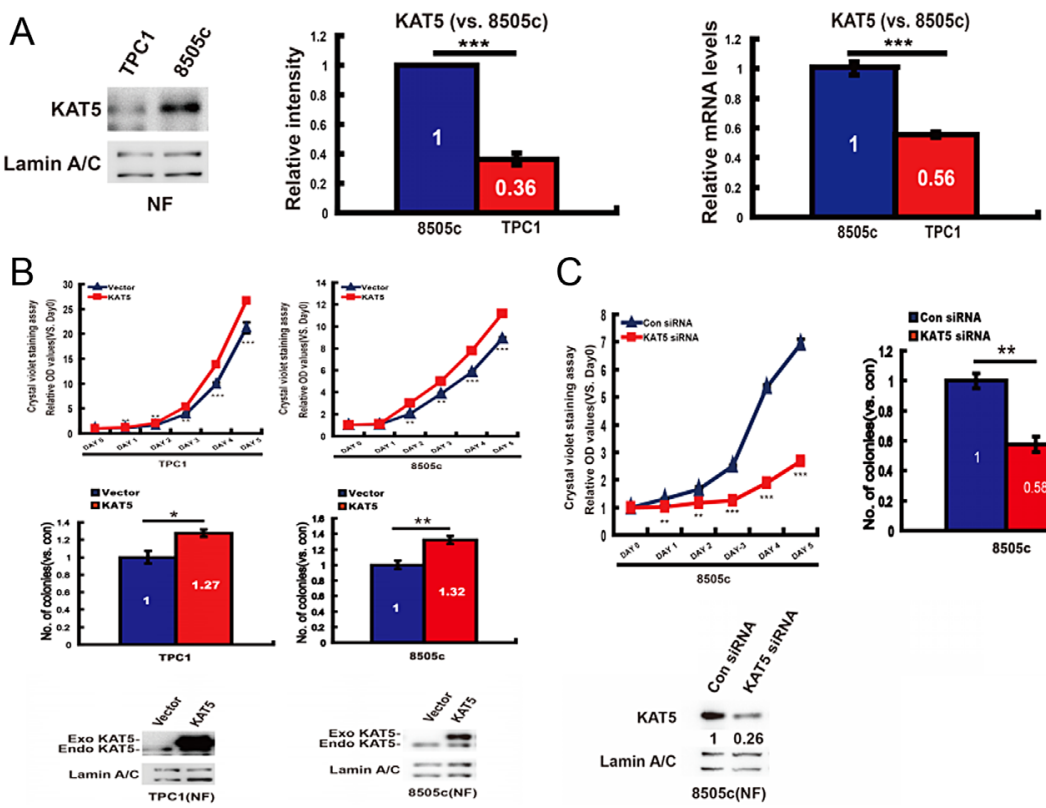

C
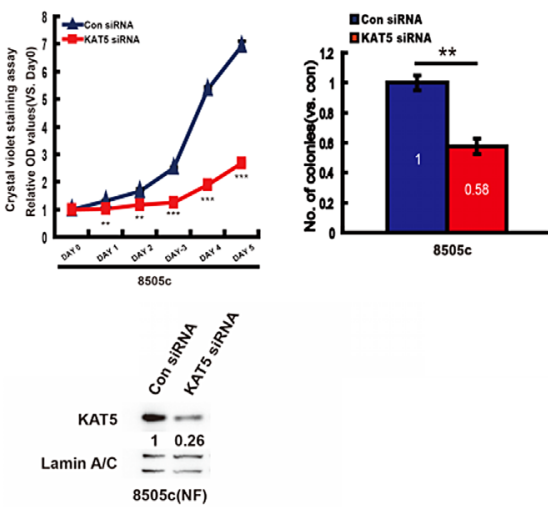

Figure 2

KAT5 promotes proliferation in thyroid cancer cells. (A) KAT5 expression detected by Western blot analysis in $8505 \mathrm{C}$ and TPC1 cells. KAT5 expression was quantified by ImageJ analysis. KAT5 mRNA was detected by RT-PCR. (B) Exogenous KAT5 was transiently expressed in TPC1 and 8505C cells detected by Western blot analysis (lower panel). Cell proliferation was detected by crystal violet staining (upper panel) and colony formation assay (middle panel). (C) 8505C cells transiently transfected with either control or KAT5 siRNA. The knockdown effect was detected by Western blot analysis (lower panel). Crystal violet staining (upper left) and the colony formation assay (upper right) were utilized to assess proliferation. Statistical significance was presented as $* P \leq 0.05, * * P \leq 0.01$ or $* * * P \leq 0.001$. A full colour version of this figure is available at https://doi.org/10.1530/ERC-18-0193. https://erc.bioscientifica.com

https://doi.org/10.1530/ERC-18-0193
(C) 2019 Society for Endocrinology Published by Bioscientifica Ltd. Printed in Great Britain 
A
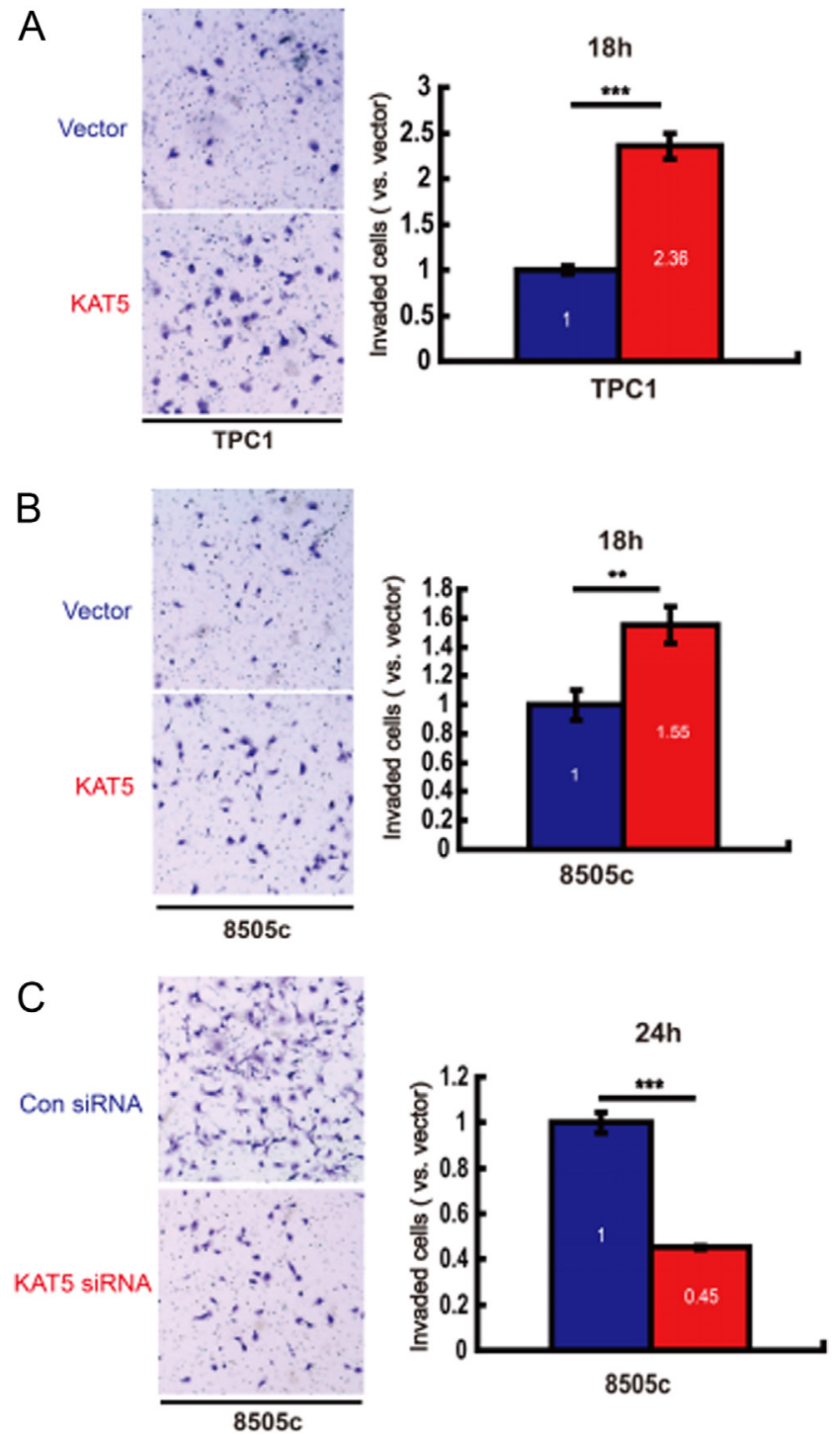

Figure 3

KAT5 promotes invasion of $8505 \mathrm{C}$ and TPC1 cells. Exogenous KAT5 was transiently expressed in TPC1 (A) and $8505 \mathrm{C}$ (B) cells, and the invasion index was measured by transwell assay. (C) $8505 \mathrm{C}$ cells were transiently transfected with control or KAT5 SiRNA, and the invasion index was measured by a transwell assay. Statistical significance was presented as $\star P \leq 0.05, * * P \leq 0.01$ or $* * * P \leq 0.001$

\section{Genetically enhanced KAT5 expression promotes thyroid cancer cell invasion}

To explore whether altered KAT5 expression is associated with ATC invasion, we performed a transwell invasion assay in TPC1and 8505C cells expressing KAT5. We found that upregulation of KAT5 significantly enhanced the invasion ability in both cells (Fig. 3A and B). Downregulation of KAT5 by siRNA remarkably inhibited invasion in 8505C cells (Fig. 3C). Using MTT and colony formation assays, we also found that upregulation of KAT5 caused resistance of the cell lines to DNA damaging agents such as doxorubicin (Fig. 4A and B) and radiotherapy (Fig. 4C and D), while downregulation of KAT5 sensitizes cell lines to the treatment (Fig. $4 \mathrm{E}$ and F). Together, these data demonstrate that KAT5 promotes proliferation and invasion and it causes resistance to radiotherapy and chemotherapy in thyroid cancer cells.

\section{KAT5 regulates C-MYC expression in thyroid cancer cells}

Because C-MYC is the major driver of cellular proliferation and invasion (Dang et al. 2009), we aimed at determining whether in thyroid cancer cells KAT5 regulated C-MYC expression. We found that C-MYC expression significantly increased in TPC1 (Fig. 5A) and 8505C (Fig. 5B) cells after forced KAT5 expression. Similarly, C-MYC expression was remarkably decreased in $8505 \mathrm{C}$ cells when KAT5 was knocked down (Fig. 5C). The inhibitory effect of KAT5 knockdown on proliferation and invasion of $8505 \mathrm{C}$ cells was completely rescued by knocking down of C-MYC (Fig. 5D, E, F and G).

\section{KAT5 inhibits C-MYC ubiquitination through acetylation}

The C-MYC protein level is mainly controlled by the ubiquitin-proteasome system (UPS) (Dang et al. 2006). To test whether KAT5 affect ubiquitin-proteasome mediated C-MYC degradation, we utilized the proteasome inhibitor MG132 to inhibit the activity of proteasome. We found that MG132 treatment blocked the KAT5-dependent regulation of C-MYC protein expression in both cell lines (Fig. 6A).

Since KAT5 acetylates histones, transcriptional factors or other non-histone proteins (Squatrito et al. 2006), we tested whether C-MYC was a substrate of KAT5 in thyroid cancer cells. With immunoprecipitation of a panacetylated antibody and western blotting C-MYC, we found KAT5 expression significantly increased acetylated C-MYC protein in both $8505 \mathrm{C}$ and TPC1 cells (Fig. 6B, C and D). To test whether KAT5 histone acetylation activity might also be affected, we also measured histone $\mathrm{H} 4$ $\mathrm{K} 5, \mathrm{~K} 8, \mathrm{~K} 12$ and $\mathrm{K} 16$ acetylation. We found no changes of total $\mathrm{H} 4$ levels but decreased AcH4 levels in KAT5 shRNA-knockdown cells compared to that of the control (Supplementary Fig. 3), indicating that in addition to C-MYC; histone acetylation is also reduced when KAT5 is knocked down.

Next, we tested the effect of KAT5 expression on C-MYC protein stability. We used cycloheximide (CHX) to 

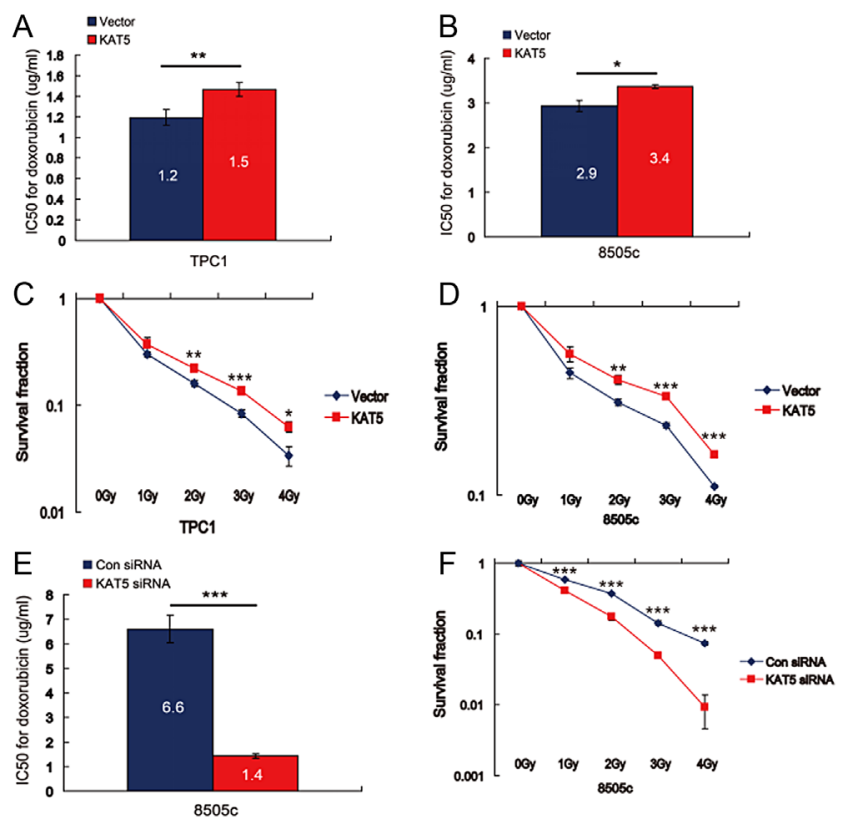

\section{Figure 4}

Expression of KAT5 reduces chemo- and radio- sensitivity. TPC1 (A) and 8505C (B) cells were transiently transfected with the vector or KAT5 plasmid, and IC50 values for doxorubicin treatment were measured by the MTT assay $48 \mathrm{~h}$ after treatment. Radiosensitivity was measured by the colony formation assay in TPC1 (C) and 8505C (D) cells. (E and F) 8505C cells were transfected with the control or KAT5 siRNA followed by doxorubicin treatment, and IC50 was measured by the MTT assay (E) and radiosensitivity was measured by the colony formation assay (F). Statistical significance was presented as $* P \leq 0.05, * * P \leq 0.01$ or $\star \star \star P \leq 0.001$. A full colour version of this figure is available at https://doi. org/10.1530/ERC-18-0193.

block protein synthesis after transfection. We found that KAT5 expression significantly increased the C-MYC halflife (Fig. 6E and F), while KAT5 knockdown dramatically decreased C-MYC half-life in 8505C cells (Fig. 6G).

Since ubiquitination and acetylation are two competing protein modifications that both occur at lysine residues, next we detected whether KAT5 regulated C-MYC ubiquitination. We co-transfected an HA-tagged ubiquitin (HA-UB) plasmid with a KAT5 expressing plasmid, and the cells were treated with MG132. We found that KAT5 overexpression significantly decreased ubiquitinated C-MYC protein level in both TPC1 (Fig. 6H) and 8505C (Fig. 6I) cells. In $8505 \mathrm{C}$ cells, we co-transfected with HA-UA, control orKAT5 siRNA and Flag-tagged C-MYC. The results showed that KAT5 knock-down remarkably increased the ubiquitinated C-MYC protein level (Fig. 6J). Taken together, our data demonstrate that KAT5-mediated acetylation stabilizes C-MYC via inhibition of UPS.
A

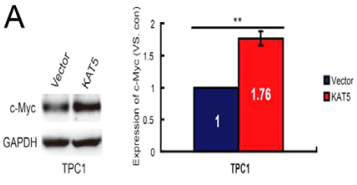

B

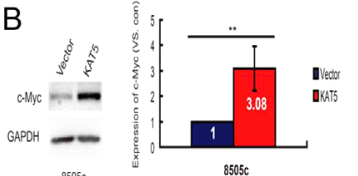

$\mathrm{F}$
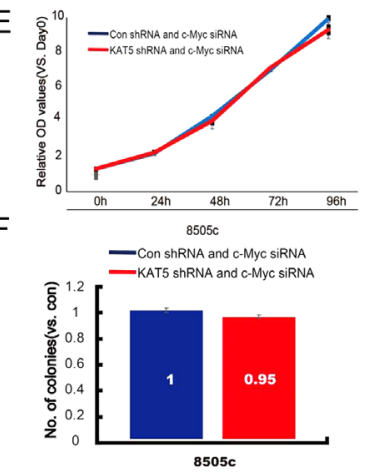

G

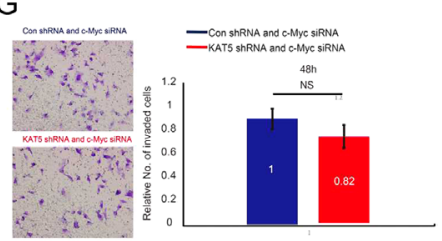

Figure 5

KAT5 enhances C-MYC expression. TPC1 (A) and 8505C (B) cells were transiently transfected with the vector or KAT5 plasmid, or 8505 C cells (C) transfected with control or KAT5 siRNA. Forty-eight hours after transfection, whole cell lysates were extracted, and C-MYC protein expression was analyzed by Western blotting. $8505 \mathrm{C}$ cells and KAT5 shRNA 8505 C cells were transfected with the control or C-MYC siRNA (C-MYC siRNA3 was used for the following experiments) (D), and IC50 was measured by the MTT assay (E) and cell proliferation was measured by the colony formation assay (F) and cell invasion was analyzed by the transwell invasion assay (G). Protein expression was quantified by Image J. Statistical significance was presented as $* P \leq 0.05, * \star P \leq 0.01$ or $* * * P \leq 0.001$. NS, not significant.

\section{Genetic modification of KAT5 expression controls thyroid cancer metastases in vivo}

To test the functional significance of KAT5-mediated C-MYC stabilization in vivo, we generated isogenic 8505C cell lines expressing shRNA control or KAT5 shRNA, and a TPC1 cell line with KAT5 overexpression (Fig. 7A and B). These stably infected cells were injected into BALB/C nude mice to generate a lung metastases model in vivo. We found that TPC1 cells expressing exogenous KAT5 displayed a higher lung metastasis rate when compared with those with the vector control $(P=0.047)$ (Fig. 7C, E and G). Meanwhile, 8505C cells with KAT5 shRNA knockdown showed a significantly fewer lung metastatic foci than those with the shRNA control $(P=0.0001)$ (Fig. 7D, F and $\mathrm{H}$ ). These results strongly support the notion that overexpression of KAT5 promotes ATC metastasis. 
A

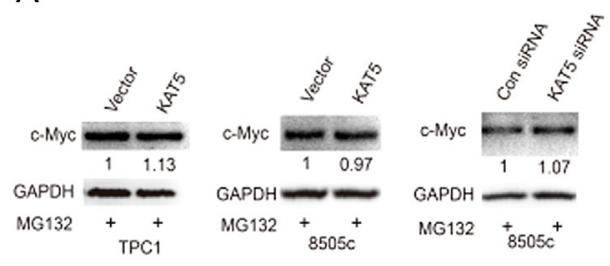

B

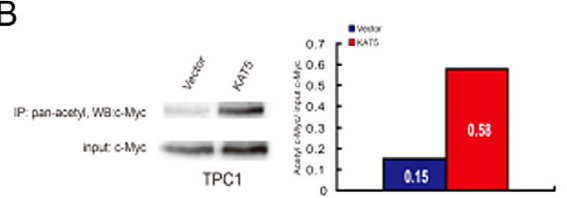

C
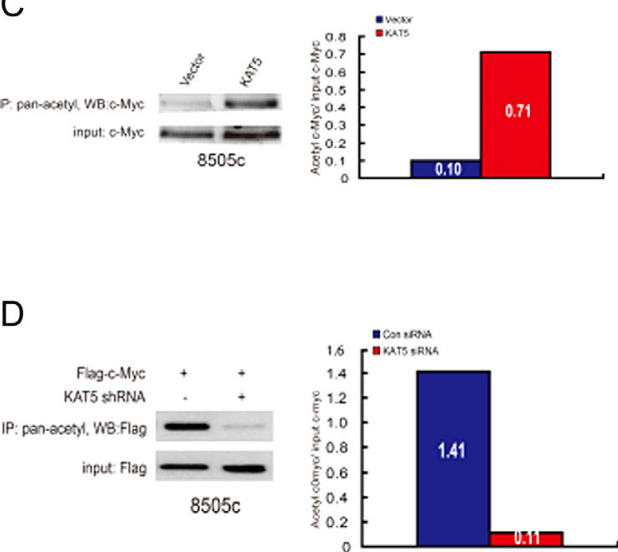

E

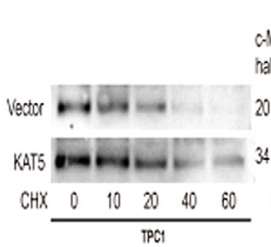

F

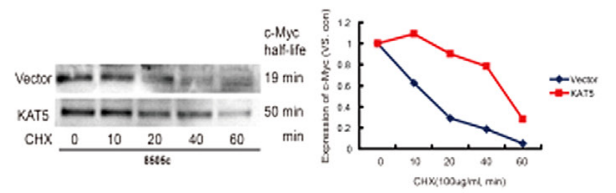

G
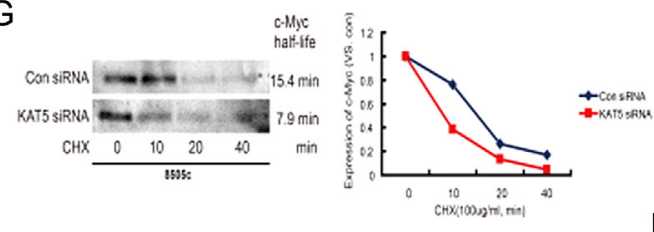

$\mathrm{H}$

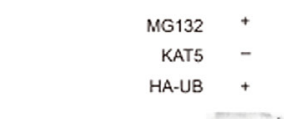

IP:HA, WB:C-Myc

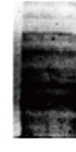

$-250 \mathrm{kDa}$

$-170 \mathrm{kDa}$

$-130 \mathrm{kDa}$

$-70 \mathrm{kDa}$

INPUT:C-MYC

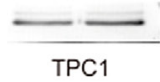

I
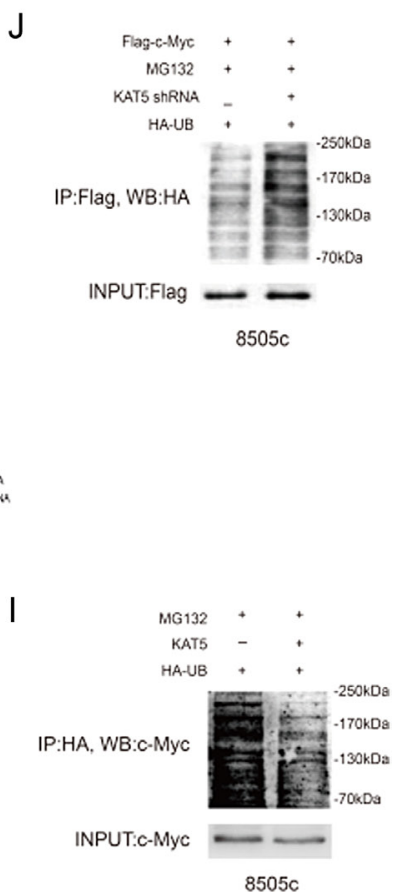

Figure 6

KAT5 mediated C-MYC acetylation inhibits ubiquitination. (A) TPC1 and 8505C cells were transiently transfected with vector or KAT5 plasmid, or 8505C cells were transfected with control or KAT5 siRNA. $48 \mathrm{~h}$ after transfection, whole cell lysates were extracted followed by Western blotting using indicated antibodies. (B, C and D) Acetylated proteins were immunoprecipitated by an anti-pan-acetyllysine antibody. The precipitates and input lysates were resolved by SDS-PAGE and probed by a C-MYC antibody. (E, F and G) TPC1 and 8505C cells were transiently transfected with the vector or KAT5 plasmid, or $8505 \mathrm{C}$ cells were transfected with control or KAT5 siRNA. Cells were treated with CHX $(100 \mu \mathrm{g} / \mathrm{mL})$ for indicated times, and C-MYC protein levels were analyzed by Western blotting. The half-life values were determined by densitometric quantitation using Image J. (H and I) TPC1 (H) and $8505 \mathrm{C}$ (I) cells were transiently transfected with HA-UB. $36 \mathrm{~h}$ after transfection, cells were treated with MG132 $(50 \mu \mathrm{g} / \mathrm{mL})$ for $4 \mathrm{~h}$. HA proteins were immunoprecipitated. The immunoprecipitates and input lysates were resolved by SDS-PAGE and probed for C-MYC antibody. (J) 8505C cells were co-transfected with HA-UB, Flag-C-MYC along with control or KAT5 siRNA. $72 \mathrm{~h}$ after transfection, cells were treated with MG132 $(50 \mu \mathrm{g} / \mathrm{mL})$ for $4 \mathrm{~h}$, and then proteins were extracted, flag tagged proteins were immunoprecipitated. The immunoprecipitates and input lysates were resolved by SDS-PAGE and were probed for anti-HA antibody. A full colour version of this figure is available at https://doi.org/10.1530/ERC-18-0193.

\section{Discussion}

Although ATC only accounts for approximately $2 \%$ of all thyroid carcinoma, it causes the majority of thyroid carcinoma-related death (Xing et al. 2013). Therefore, investigating the molecular mechanisms driving ATC progression, metastasis and resistance to treatment has significant impact on identification of novel therapeutic targets for better clinical treatment. In this report, we provide the clinical, in vitro and in vivo evidence highlighting the functional significance of the DDR protein, KAT5, in which overexpression leads to enhanced ATC invasiveness via regulation of C-MYC.
Owing to the rarity of ATC cases in a single clinical center, it has been difficult to study biomarkers as well as mechanisms for ATC. Tianjin Medical University Cancer Hospital has one of the largest thyroid cancer departments in the world. With more than 7000 thyroid cancer surgeries each year, the entity has accumulated a handful of ATC cases that allow for biomarker and mechanistic studies. In this study, we started with bioinformatics analysis with the TCGA database and identified KAT5 as a hub protein of many of the mutated or abnormally regulated DDR proteins. We assessed KAT5 expression in samples from 82 ATC cases, which accounts for the largest number of clinical cases to date from a single 
A
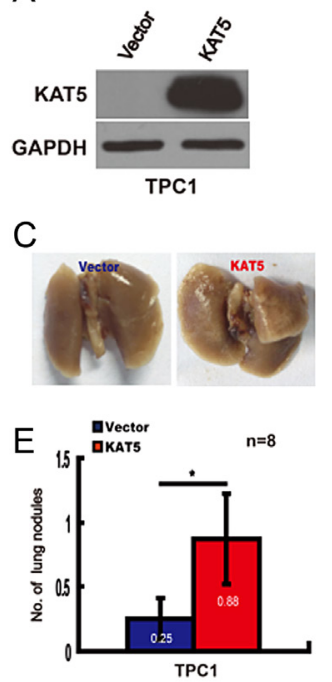

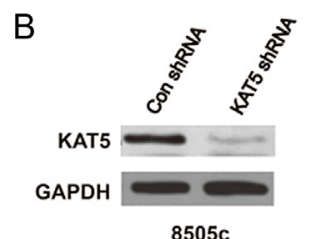

D
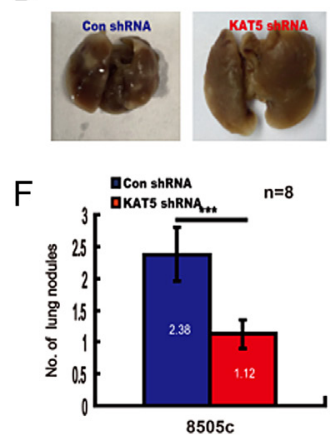

G

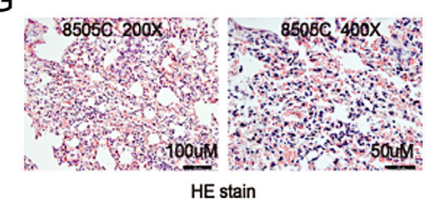

$\mathrm{H}$

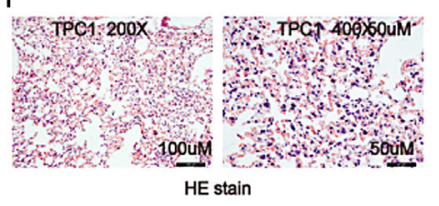

Figure 7

Genetic modification of KAT5 impacts thyroid cancer metastases in vivo. TPC1 (A) was stably transfected with vector or KAT5, and $8505 \mathrm{C}$ cells (B) were infected with control and KAT5 shRNA, respectively. Whole cell lysates from the pooled cells were collected for protein analysis by Western blot. The stable cells were injected via the tail vein into the BALB/c nude mice. Ten weeks after injection, mice were killed and lungs were removed. Lung metastasis was measured by counting metastatic foci in the lungs.

Representative photographs of the lung are shown in (C) and (D), and the number of lung metastases foci was plotted in (E) and (F). The representative hematoxylin-eosin (HE)- stained images of lung metastatic foci were shown in (G) and $(\mathrm{H})$ (Scale bar: $100 \mu \mathrm{m}$ and $50 \mu \mathrm{m}$ ). Statistical significance was presented as $* P \leq 0.05$, $\star \star P \leq 0.01$, or $* \star \star P \leq 0.001$. cancer center. Our results demonstrate that higher KAT5 level correlates with metastasis and poorer survival rate in ATC. We also studied the mechanisms of KAT5-mediated invasiveness via C-MYC acetylation, and the results were further supported by the in vivo analysis showing that KAT5 mediates lung metastases in the ATC xenograft animal model.

KAT5 is a lysine acetyltransferase, and it has been shown as a tumor suppressor in several cancers, including breast cancer, lung cancer, glioblastoma and melanoma (Sun et al. 2010). However, it has also been reported that it is up-regulated and associated with oncoprotein properties in malignant pleural mesothelioma and prostate cancer (Shiota et al. 2010, Cregan et al. 2016). On a broader picture of DDR pathways, earlier clinical data have demonstrated that DDR is an antitumor barrier during early tumorigenesis, echoing its cellular function to maintain genome stability (Bartek et al. 2007a). However, there is growing evidence that DDR elements might play a role in tumor invasion and metastasis. The ATM kinase, for example, has been shown to regulate Snail stability via phosphorylation of Serine 100, hence promoting invasion and metastasis in breast cancer (Sun et al. 2012, Boohaker et al. 2013). Many DDR proteins have been added to the list of contributing factors to tumor metastasis and resistance to DNA damaging agents, when activated and/ or overexpressed (Broustas \& Lieberman 2014). Our data from in vitro, in vivo and clinical data strongly support the notion that elevated KAT5 expression promotes ATC invasion and metastasis, highlighting direct evidence of a novel mechanism for ATC invasiveness.

It is still unclear what might drive elevation of KAT5 in ATC. We suspect that tumor microenvironment might play a significant role in the process. Chronic inflammation, for example, frequently found in ATC (Suzuki et al. 2013), might contribute to upregulation of KAT5. Additionally, in ATC tissues there are much higher numbers of mitotic cells (Smith \& Nucera 2015), a cell division stage where many of the DDR genes are activated, and thus potentially causing KAT5 upregulation.

The transcription factor C-MYC is one of the most frequently overexpressed oncogenes in cancers, as this classic oncoprotein plays a pivotal role in tumorigenesis and metastasis (Dang et al. 2009). The expression level of C-MYC is significantly higher in ATC cells (Wagle et al. 2014, Uruno et al. 2015, Enomoto et al. 2017). Our data provide a mechanistic insight into C-MYC upregulation. Our biochemical analyses in the in vitro system demonstrate that KAT5 regulates C-MYC protein expression through post translational modification, including acetylation and hence inhibition of UPS. A study showing that C-MYC is acetylated by KAT5 further supports this notion (Patel et al. 2004).

With KAT5 being an upstream regulator of C-MYC, it is likely that KAT5 inhibitors may abrogate the invasiveness of ATC. Indeed, a KAT5 inhibitor NU9056 has been shown to inhibit protein acetylation and decrease proliferation of prostate cancer cells (Coffey et al. 2012). Those inhibitors are also likely to enhance the sensitivity of chemotherapy and radiotherapy. We have provided the proof of principal that knocking down KAT5 leads to reduced invasion/ metastasis and enhanced radiosensitivity both in vitro and in vivo. Future development of KAT5 inhibitors is likely to significantly benefit APC patients.

In conclusion, our data presented here provide a novel molecular mechanism driving ATC invasiveness, 
metastasis and resistance to chemotherapy and radiotherapy.

\section{Supplementary data}

This is linked to the online version of the paper at https://doi.org/10.1530/ ERC-18-0193.

\section{Declaration of interest}

The authors declare that there is no conflict of interest that could be perceived as prejudicing the impartiality of the research reported.

\section{Funding}

This work was supported in part by the following research grants: the fund (2016YFC0904600) from the Chinese Ministry of Science and Technology, US NIH R01CA133093, the Alabama Innovation Fund, the National Natural Science Foundation of China (81672743, 81771852, 81401412, 81372411) and a fellowship from the China Oversea Scholarship Council and Suzhou Science and Education Project (No. KJXW2017010).

\section{Author contribution statement}

Experiments were conceived and designed by $X \mathrm{~W}, \mathrm{M} G$ and $B \mathrm{X}$. Experiments and data analysis were performed by $X W, S C, R B, Y L, B L, S$ $Z, Y T$. Overall discussions of the data and implications involved all authors; and the manuscript was written by X W, S C and B X.

\section{Acknowledgements}

The authors thank Dr Michael Xing (Johns Hopkins University) for providing the TPC1 cell line. The KAT5-pcDNA HisMax plasmid was kindly provided by Prof. Yuet-kin Leung (University of Cincinnati College of Medicine). They thank Dr Lynae Hanks (Southern Research Institute) for proof reading of the manuscript.

\section{References}

Abdulghani J, Gokare P, Gallant JN, Dicker D, Whitcomb T, Cooper T, Liao JG, Derr J, Liu J, Goldenberg D, et al. 2016 Sorafenib and quinacrine target anti-apoptotic protein MCL1: a poor prognostic marker in anaplastic thyroid cancer (ATC). Clinical Cancer Research 22 6192-6203. (https://doi.org/10.1158/1078-0432.CCR-15-2792)

Bartek J, Bartkova J \& Lukas J $2007 a$ DNA damage signalling guards against activated oncogenes and tumour progression. Oncogene $\mathbf{2 6}$ 7773-7779. (https://doi.org/10.1038/sj.onc.1210881)

Bartek J, Lukas J \& Bartkova J 2007b DNA damage response as an anticancer barrier: damage threshold and the concept of 'conditional haploinsufficiency'. Cell Cycle 6 2344-2347. (https://doi.org/10.4161/ cc.6.19.4754)

Bhoumik A, Singha N, O'Connell MJ \& Ronai ZA 2008 Regulation of TIP60 by ATF2 modulates ATM activation. Journal of Biological Chemistry 283 17605-17614. (https://doi.org/10.1074/jbc. M802030200)

Boohaker RJ \& Xu B 2014 The versatile functions of ATM kinase. Biomedical Journal 37 3-9. (https://doi.org/10.4103/23194170.125655)
Boohaker RJ, Cui XL, Stackhouse M \& Xu B 2013 ATM-mediated snail serine 100 phosphorylation regulates cellular radiosensitivity. Radiotherapy and Oncology 108 403-408. (https://doi.org/10.1016/j. radonc.2013.06.017)

Broustas CG \& Lieberman HB 2014 DNA damage response genes and the development of cancer metastasis. Radiation Research $\mathbf{1 8 1}$ 111-130. (https://doi.org/10.1667/RR13515.1)

Coffey K, Blackburn TJ, Cook S, Golding BT, Griffin RJ, Hardcastle IR, Hewitt L, Huberman K, Mcneill HV, Newell DR, et al. 2012 Characterisation of a Tip60 specific inhibitor, NU9056, in prostate cancer. PLOS ONE 7 e45539. (https://doi.org/10.1371/journal. pone.0045539)

Cregan S, Mcdonagh L, Gao Y, Barr MP, O’Byrne KJ, Finn SP, Cuffe S \& Gray SG 2016 KAT5 (Tip60) is a potential therapeutic target in malignant pleural mesothelioma. International Journal of Oncology 48 1290-1296. (https://doi.org/10.3892/ijo.2016.3335)

Dang CV, O’Donnell KA, Zeller KI, Nguyen T, Osthus RC \& Li F 2006 The C-MYC target gene network. Seminars in Cancer Biology 16 253-264. (https://doi.org/10.1016/j.semcancer.2006.07.014)

Dang CV, Le A \& Gao P 2009 MYC-induced cancer cell energy metabolism and therapeutic opportunities. Clinical Cancer Research 15 6479-6483. (https://doi.org/10.1158/1078-0432.CCR-09-0889)

Enomoto K, Zhu XG, Park S, Zhao L, Zhu YL J., Willingham MC, Qi J, Copland JA, Meltzer P \& Cheng SY 2017 Targeting MYC as a therapeutic intervention for anaplastic thyroid cancer. Journal of Clinical Endocrinology and Metabolism 102 2268-2280. (https://doi. org/10.1210/jc.2016-3771)

Gorrini C, Squatrito M, Luise C, Syed N, Perna D, Wark L, Martinato F, Sardella D, Verrecchia A, Bennett S, et al. 2007 Tip60 is a haploinsufficient tumour suppressor required for an oncogene-induced DNA damage response. Nature 448 1063-1067. (https://doi. org/10.1038/nature06055)

Guerra A, Di Crescenzo V, Garzi A, Cinelli M, Carlomagno C, Tonacchera M, Zeppa P \& Vitale M 2013 Genetic mutations in the treatment of anaplastic thyroid cancer: a systematic review. BMC Surgery 13 (Supplement 2) S44. (https://doi.org/10.1186/1471-248213-S2-S44)

Hanahan D \& Weinberg RA 2011 Hallmarks of cancer: the next generation. Cell 144 646-674. (https://doi.org/10.1016/j. cell.2011.02.013)

Kaidi A \& Jackson SP 2013 KAT5 tyrosine phosphorylation couples chromatin sensing to ATM signalling. Nature 498 70-74. (https:// doi.org/10.1038/nature12201)

Kitahara CM \& Sosa JA 2016 The changing incidence of thyroid cancer Nature Reviews Endocrinology 12 646-653. (https://doi.org/10.1038/ nrendo.2016.110)

Lord CJ \& Ashworth A 2012 The DNA damage response and cancer therapy. Nature 481 287-294. (https://doi.org/10.1038/nature10760)

Molinaro E, Romei C, Biagini A, Sabini E, Agate L, Mazzeo S, Materazzi G, Sellari-Franceschini S, Ribechini A, Torregrossa L, et al. 2017 Anaplastic thyroid carcinoma: from clinicopathology to genetics and advanced therapies. Nature Reviews Endocrinology 13 644-660. (https://doi.org/10.1038/nrendo.2017.76)

Pandey AK, Zhang YZ, Zhang ST, Li Y, Tucker-Kellogg G, Yang H \& Jha S 2015 TIP60-miR-22 axis as a prognostic marker of breast cancer progression. Oncotarget 6 41290-41306. (https://doi.org/10.18632/ oncotarget.5636)

Patel JH, Du YP, Ard PG, Phillips C, Carella B, Chen CJ, Rakowski C, Chatterjee C, Lieberman PM, Lane WS, et al. 2004 The C-MYC oncoprotein is a substrate of the acetyltransferases hGCN5/PCAF and TIP60. Molecular and Cellular Biology 24 10826-10834. (https://doi. org/10.1128/MCB.24.24.10826-10834.2004)

Shiota M, Yokomizo A, Masubuchi D, Tada Y, Inokuchi J, Eto M, Uchiumi T, Fujimoto N \& Naito S 2010 Tip60 promotes prostate cancer cell proliferation by translocation of androgen receptor into c) 2019 Society for Endocrinology Published by Bioscientifica Ltd. Printed in Great Britain 
the nucleus. Prostate 70 540-554. (https://doi.org/10.1002/ pros.21088)

Smallridge RC, Marlow LA \& Copland JA 2009 Anaplastic thyroid cancer: molecular pathogenesis and emerging therapies. EndocrineRelated Cancer 16 17-44. (https://doi.org/10.1677/ERC-08-0154)

Smith N \& Nucera C 2015 Personalized therapy in patients with anaplastic thyroid cancer: targeting genetic and epigenetic alterations. Journal of Clinical Endocrinology and Metabolism 100 35-42. (https://doi.org/10.1210/jc.2014-2803)

Squatrito M, Gorrini C \& Amati B 2006 Tip60 in DNA damage response and growth control: many tricks in one HAT. Trends in Cell Biology 16 433-442. (https://doi.org/10.1016/j.tcb.2006.07.007)

Sun Y, Jiang X, Chen S, Fernandes N \& Price BD 2005 A role for the Tip60 histone acetyltransferase in the acetylation and activation of ATM. PNAS 102 13182-13187. (https://doi.org/10.1073/ pnas.0504211102)

Sun Y, Xu Y, Roy K \& Price BD 2007 DNA damage-induced acetylation of lysine 3016 of ATM activates ATM kinase activity. Molecular and Cellular Biology 27 8502-8509. (https://doi.org/10.1128/ MCB.01382-07)

Sun Y, Jiang X, Xu Y, Ayrapetov MK, Moreau LA, Whetstine JR \& Price BD 2009 Histone H3 methylation links DNA damage detection to activation of the tumour suppressor Tip60. Nature Cell Biology 11 1376-1382. (https://doi.org/10.1038/ncb1982)

Sun Y, Jiang X \& Price BD 2010 Tip60: connecting chromatin to DNA damage signaling. Cell Cycle 9 930-936. (https://doi.org/10.4161/ cc.9.5.10931)

Sun MN, Guo XJ, Qian XL, Wang HB, Yang CY, Brinkman KL, SerranoGonzalez M, Jope RS, Zhou BH, Engler DA, et al. 2012 Activation of the ATM-Snail pathway promotes breast cancer metastasis. Journal of Molecular Cell Biology 4 304-315. (https://doi.org/10.1093/jmcb/mjs048)

Sun YF, Sun JW, Lungchukiet P, Quarni W, Yang SY, Zhang XH \& Bai WL 2015 Fe65 suppresses breast cancer cell migration and invasion through Tip60 mediated cortactin acetylation. Scientific Reports 5 11529. (https://doi.org/10.1038/srep11529)

Suzuki S, Shibata M, Gonda K, Kanke Y, Ashizawa M, Ujiie D, Suzushino S, Nakano K, Fukushima T, Sakurai K, et al. 2013 Immunosuppression involving increased myeloid-derived suppressor cell levels, systemic inflammation and hypoalbuminemia are present in patients with anaplastic thyroid cancer. Molecular and Clinical Oncology 1 959-964. (https://doi.org/10.3892/mco.2013.170)

Takino T, Nakada M, Li Z, Yoshimoto T, Domoto T \& Sato H 2016 Tip60 regulates MT1-MMP transcription and invasion of glioblastoma cells through NF-kappaB pathway. Clinical and Experimental Metastasis 33 45-52. (https://doi.org/10.1007/s10585-015-9756-8)

Uruno T, Masaki C, Akaishi J, Matsuzu K, Suzuki A, Ohkuwa K, Shibuya H, Kitagawa W, Nagahama M, Sugino K \& Ito K 2015 Chemosensitivity of anaplastic thyroid cancer based on a histoculture drug response assay. International Journal of Endocrinology 2015 967286. (https://doi.org/10.1155/2015/967286)

Wagle N, Grabiner BC, Van Allen EM, Amin-Mansour A, TaylorWeiner A, Rosenberg M, Gray N, Barletta JA, Guo YN, Swanson SJ, et al. 2014 Response and acquired resistance to everolimus in anaplastic thyroid cancer. New England Journal of Medicine 371 1426-1433. (https://doi.org/10.1056/NEJMoa1403352)

Wei X, Li Y, Zhang S \& Ming G 2014 Evaluation of thyroid cancer in Chinese females with breast cancer by vascular endothelial growth factor (VEGF), microvessel density, and contrast-enhanced ultrasound (CEUS). Tumor Biology 35 6521-6529. (https://doi. org/10.1007/s13277-014-1868-2)

Xing M 2013 Molecular pathogenesis and mechanisms of thyroid cancer. Nature Reviews Cancer 13 184-199. (https://doi.org/10.1038/ $\operatorname{nrc} 3431)$

Xing M, Haugen BR \& Schlumberger M 2013 Progress in molecularbased management of differentiated thyroid cancer. Lancet $\mathbf{3 8 1}$ 1058-1069. (https://doi.org/10.1016/S0140-6736(13)60109-9)
Received in final form 1 September 2018

Accepted 19 September 2018
(C) 2019 Society for Endocrinology Published by Bioscientifica Ltd. Printed in Great Britain 